\title{
Diagnosing Cysts With Correlation Coefficient Images From 2-Dimensional Freehand Elastography
}

\author{
Rebecca C. Booi, PhD, Paul L. Carson, PhD, Matthew O'Donnell, PhD, \\ Michael S. Richards, PhD, Jonathan M. Rubin, MD, PhD
}

\begin{abstract}
Objective. We compared the diagnostic potential of using correlation coefficient images versus elastograms from 2-dimensional (2D) freehand elastography to characterize breast cysts. Methods. In this preliminary study, which was approved by the Institutional Review Board and compliant with the Health Insurance Portability and Accountability Act, we imaged 4 consecutive human subjects (4 cysts, 1 biopsy-verified benign breast parenchyma) with freehand 2D elastography. Data were processed offline with conventional 2D phase-sensitive speckle-tracking algorithms. The correlation coefficient in the cyst and surrounding tissue was calculated, and appearances of the cysts in the correlation coefficient images and elastograms were compared. Results. The correlation coefficient in the cysts was considerably lower (14\%-37\%) than in the surrounding tissue because of the lack of sufficient speckle in the cysts, as well as the prominence of random noise, reverberations, and clutter, which decorrelated quickly. Thus, the cysts were visible in all correlation coefficient images. In contrast, the elastograms associated with these cysts each had different elastographic patterns. The solid mass in this study did not have the same high decorrelation rate as the cysts, having a correlation coefficient only $2.1 \%$ lower than that of surrounding tissue. Conclusions. Correlation coefficient images may produce a more direct, reliable, and consistent method for characterizing cysts than elastograms. Key words: breast; correlation; cyst; elasticity imaging; elastography; speckle tracking.
\end{abstract}

Abbreviations

2D, 2-dimensional

Received March 1, 2007, from the Departments of Radiology (R.C.B., P.L.C., M.S.R., J.M.R.) and Biomedical Engineering (R.C.B., P.L.C., M.O.), University of Michigan, Ann Arbor, Michigan USA; and Department of Biomedical Engineering, University of Washington, Seattle, Washington USA (M.O.). Revision requested April 10, 2007. Revised manuscript accepted for publication May 17, 2007.

This work was supported in part by National Institutes of Health grants R21-CA109440 and R01CA091713. Dr Booi and Dr Carson are supported by a federal grant in conjunction with General Electric Company.

Address correspondence to Jonathan M. Rubin, MD, PhD, University of Michigan, 1500 E Medical Center Dr, Ann Arbor, MI 48109-0553 USA.

E-mail: jrubin@umich.edu

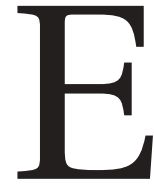

lasticity imaging, also known as elastography, has consistently shown potential for improving diagnosis of diseases in many parts of the body, including the cardiovascular system, ${ }^{1-3}$ kidney, ${ }^{4}$ deep venous thrombosis in the lower extremities, ${ }^{5}$ prostate, ${ }^{6,7}$ breast, ${ }^{8-10}$ and thyroid. ${ }^{11}$ However, recent studies attempting to characterize cysts with elastograms have had limited, and sometimes conflicting, results. Using a Diasonics (Santa Clara, CA) ultrasound scanner, Garra et $\mathrm{al}^{8}$ described 3 different elastographic patterns in the 8 cysts studied: (1) a darker (stiffer) area with a brighter (softer) center, (2) a uniformly dark area, and (3) an area with both bright and dark regions. Using a Siemens Medical Solutions (Mountain View, CA) ultrasound scanner, Barr ${ }^{10}$ reported that both simple and complicated cysts appeared dark (stiffer) in elas- 
tograms with a brighter "bulls-eye" appearance in the middle of the cyst. Hall et $\mathrm{al}^{9}$ described that because cysts rapidly decorrelate, they can appear relatively stiff or soft in elastograms, often depending on how much strain is applied. In a study conducted by Lyshchik et al, ${ }^{11} 5$ of 6 cysts imaged in the thyroid produced considerable noise in their resulting elastograms, preventing the authors from identifying any elastographic characteristics of these lesions. From these findings, it is evident that cysts can have a variety of appearances in elastograms, depending on the ultrasound scanner used, percent compression and precompression, and type of signal processing.

In response to this problem, our study evaluated whether the high decorrelation rate initially observed by Hall et $\mathrm{al}^{9}$ associated with cysts in cross-correlation imaging could be used directly to distinguish cysts from solid masses. Because most cysts are primarily composed of fluid, random noise, reverberations, side lobes, and clutter, they decorrelate quickly when compressed for elastography. Therefore, cysts may be consistently and clearly visible in the correlation coefficient image, providing direct diagnostic information that cannot be achieved by looking at the appearances of cysts on elastograms.

\section{Materials and Methods}

\section{Human Subjects and Data Acquisition}

This study was approved by the Institutional Review Board, and informed consent was obtained from all human subjects. The study was compliant with the Health Insurance Portability and Accountability Act, and subject confidentiality was protected at all times.
To obtain preliminary data for this study, 4 consecutive subjects were imaged with freehand elastography conducted by an experienced physician (J.M.R.). Two subjects each had 1 clinically confirmed cyst; 1 subject had 2 clinically confirmed cysts; and 1 subject contained a biopsyproven benign breast parenchyma with prominent stromal fibrosis. Up to 91 radio frequency images, corresponding to maximum $7 \%$ compression, were acquired with a LOGIQ 9 ultrasound scanner (GE Healthcare, Milwaukee, WI). Data were processed offline.

\section{Derivation of the Correlation Coefficient From 2-Dimensional Speckle-Tracking Algorithms}

The correlation coefficient images in this study were produced from conventional 2-dimensional (2D) phase-sensitive speckle-tracking algorithms, which estimate tissue motion with correlation-based processing in the axial (along the ultrasound beam) and lateral (perpendicular to ultrasound beam) directions. ${ }^{12,13}$ Estimated displacements were accumulated in $0.3 \%$ to $0.6 \%$ compression steps. ${ }^{14}$ The gradient of the axial displacements was used to calculate strain.

To calculate the $2 \mathrm{D}$ correlation coefficient, first a 2D correlation kernel (spatially equivalent to 1 speckle spot) was defined around every pixel in the complex predeformation image. The size of the speckle in each direction was determined from the full width at half maximum of the 2D autocorrelation function of the image. Next, the kernel was cross-correlated with the complex postdeformation image. The resulting unit-normalized, complex cross-correlation coefficient $\rho^{\prime}{ }_{x y}$ at pixels $(x, y)$ as a function of lags $\left(l_{x}, l_{y}\right)$ is described in Equation 1. In this equation, $G_{t}(x, y)$ is the original predeformation image; $G_{t+1}(x, y)$ is the postdeformation image; and $W_{i j}$ is a 2D Hamming window over the 2D correlation kernel:

$$
\rho_{x y}^{\prime}\left(l_{x}, l_{y}\right)=\frac{\sum_{i} \sum_{j} W_{i j}\left[G_{t}(x+i, y+j) G_{t+1}^{*}\left(x+l_{x}+i, y+l_{y}+j\right)\right]}{\left.-\sum_{i} \sum_{j} W_{i j}\left|G_{t}(x+i, y+j)\right|^{2}\right]^{1 / 2}\left[\sum_{i} \sum_{j} W_{i j}\left|G_{t+1}^{*}\left(x+l_{x}+i, y+l_{y}+j\right)\right|^{2}\right]^{1 / 2}} .
$$


To improve the signal-to-noise ratio, the correlation coefficient was filtered with a unity gain function $\left(\sum F_{i j}=1\right)$ twice the speckle size:

$$
\text { (2) } \rho_{x y}\left(l_{x}, l_{y}\right)=\sum_{i} \sum_{j} F_{i j}\left[\rho_{x+i, y+j}^{\prime}\left(l_{x}, l_{y}\right)\right] \text {. }
$$

The 2D displacement vector was then estimated from the peak position of the complex correlation function described in Equation 2 in a 2-step process. First, both axial and lateral displacements were determined from the correlation lags, which corresponded to the maximum value of the magnitude of the correlation function. Next, the axial displacement estimate was refined using the position of the closest zero crossing of the phase of the complex correlation function. The final outputs of this processing were the lateral displacement $u(x, y)$, axial displacement $v(x, y)$, and magnitude of the correlation coefficient $|\rho(x, y)|$. Breast masses in this study were manually segmented in the original gray scale image, and the correlation coefficient in the mass was compared with the correlation coefficient outside the mass but within the $40 \times$ 39-mm (axial $\times$ lateral) image.

\section{Quantification of Mass Visibility in Images}

To quantify the high variability in the appearance of cysts in strain imaging, we calculated the strain signal-to-noise ratio, $S / N(\varepsilon)$, in the cysts. We used the standard formula

$$
S / N(\varepsilon)=\frac{<\varepsilon_{c}>}{\sigma_{\varepsilon-c}},
$$

where $\left\langle\varepsilon_{c}>\right.$ is the average strain in the cyst, and $\sigma_{e-c}$ is the SD of the strain estimates in the cyst.

For comparison, we used a corresponding formula to show the signal-to-noise ratio in the correlation coefficient images, $S / N(\rho)$ :

$$
S / N(\rho)=\frac{<\rho_{c}>}{\sigma_{\rho-c}},
$$

where $\left\langle\rho_{c}>\right.$ is the mean correlation coefficient in the cyst, and $\sigma_{\rho-c}$ is the SD of the correlation coefficients in the cyst.
In addition, to show how this high strain variation in cysts can obscure the appearance of cysts in elastograms, we compared the contrast-tonoise ratios between the strain images and correlation coefficient images of all cysts in this study. The contrast-to-noise ratio in strain images, $C / N(\varepsilon)$, is defined as

$$
C / N(\varepsilon)=\frac{\left|<\varepsilon_{c}>-<\varepsilon_{b}\right\rangle \mid}{\sqrt{\frac{\sigma_{\varepsilon-c}^{2}+\sigma_{\varepsilon-b}^{2}}{2}}},
$$

where $\left\langle\varepsilon_{c}>\right.$ and $\left\langle\varepsilon_{b}>\right.$ are the mean strains in the cyst and background, respectively; $\sigma_{\varepsilon-c}^{2}$ is the variance of the strain estimates in the cyst; $\sigma_{\varepsilon-b}^{2}$ is the variance of the strain estimates in the background; and || represents the absolute value.

A similar measurement can be performed to show the contrast-to-noise ratio, $C / N(\rho)$, between the cyst and background correlation coefficients:

$$
C / N(\rho)=\frac{\left|<\rho_{c}>-<\rho_{b}\right\rangle \mid}{\sqrt{\frac{\sigma_{\rho-c}^{2}+\sigma_{\rho-b}^{2}}{2}}},
$$

where $<\rho_{c}>$ is the mean correlation coefficient in the cyst; $\left\langle\rho_{b}\right\rangle$ is the mean correlation coefficient in the background; $\sigma_{\rho-c}^{2}$ is the variance of the correlation coefficient estimates in the cyst; $\sigma^{2}{ }_{\rho-b}$ is the variance of the correlation coefficient estimates in the background; and || represents the absolute value.

\section{Results}

Figures $1-3$ show cysts with varying elastographic appearances. Note that the strains in each of these cysts are highly variable, with high- and low-strain regions distributed throughout each cyst. This finding is consistent with the lowstrain signal-to-noise ratios in the cysts (Table 1). In contrast to the variability of cyst appearances in these elastograms, the cysts look much more uniform in the correlation coefficient images with higher signal-to-noise ratios (Table 1). For the 4 cases analyzed in this preliminary study, the signal-to-noise ratios in the cysts were at least 5 times higher in the correlation coefficient images than in the strain images. 

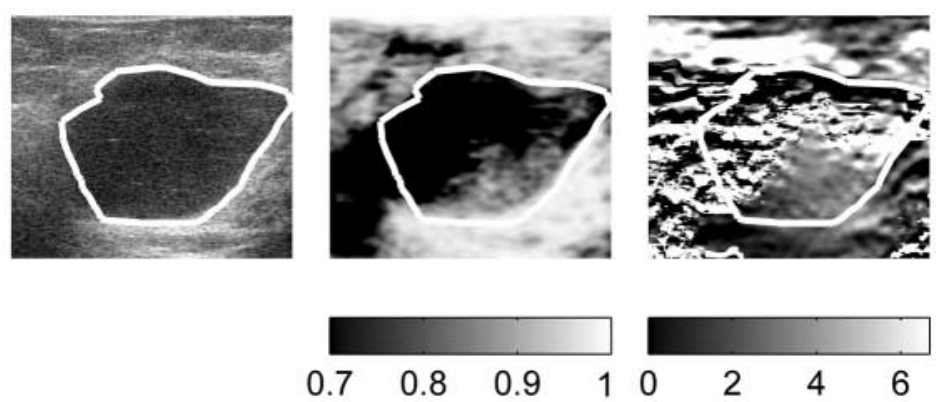

Figure 1. Gray scale image (left), correlation coefficient image (center), and elastogram (right) of a simple cyst. Black regions in the correlation coefficient image correspond to areas of poor correlation; black regions in the elastogram correspond to areas of low strain (stiffer tissue). Strains are represented in percents. The correlation coefficient image clearly shows the cyst, which has a mean correlation of 0.65 and is $28 \%$ lower than the background correlation of 0.86 . However, the elastographic area of the cyst is largely dominated by noise artifacts associated with the poor tracking capabilities of the algorithms in the cyst, obscuring cyst visibility. Original image dimensions were $38 \times 39 \mathrm{~mm}$.

Furthermore, in each of these cases, the cyst is clearly visible in the correlation coefficient image, appearing largely "black" because of its poor correlation coefficient and relatively high correlation coefficient signal-to-noise ratio. This is true even when the strain images appear mostly as noise. Specifically, the correlation coefficient values in the cysts were $14 \%$ to $36 \%$ lower than the background correlation coefficient values (Table 1). These high decorrelation rates are due

Figure 2. Gray scale image (left), correlation coefficient image (center), and elastogram (right) of a nonsimple cyst. Black regions in the correlation coefficient image correspond to areas of poor correlation; black regions in the elastogram correspond to areas of low strain (stiffer tissue). Strains are represented in percents. The mean correlation coefficient in the cyst is 0.79 and is $14 \%$ lower than the background correlation of 0.92 . Although the cyst is clearly visible in the correlation coefficient image [C/N( $\rho)=1.9]$, it is not visible in the elastogram based on a very low contrast-to-noise ratio $[C / N(\varepsilon)=0.20]$. Original image dimensions were 38 $\times 26 \mathrm{~mm}$.
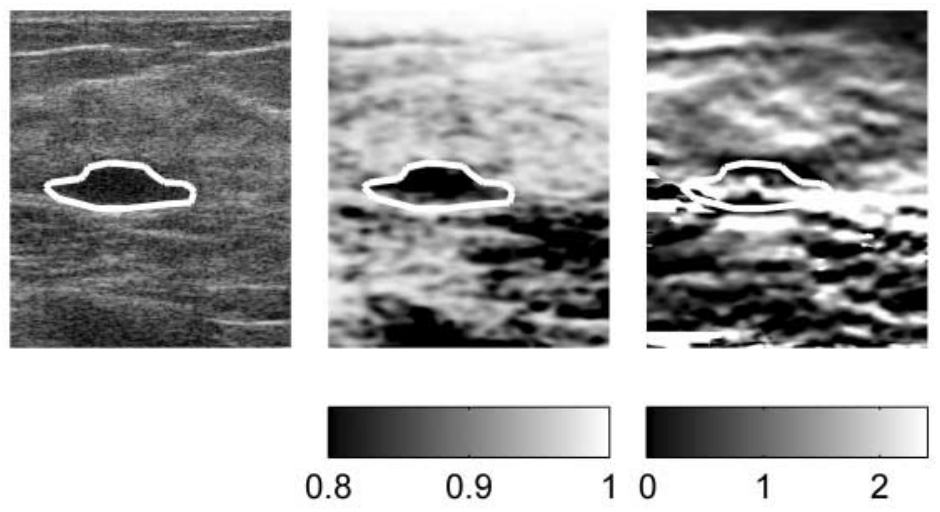

to the fact that most signal in cysts is not speckle but, rather, noise, reverberations, and clutter. The differences in the quality of the correlation coefficient images of the cysts versus the strain images are manifested in the relatively high contrast-to-noise ratios in the correlation coefficient images compared with the low contrast-to-noise ratios in the strain images. Conversely, the solid mass in Figure 4, a benign breast parenchyma with prominent stromal fibrosis, had a correlation coefficient much closer to the background value and thus a more reproducible and reliable elastogram.

\section{Discussion}

Although simple cysts are easily identified on conventional ultrasound imaging, nonsimple (complicated or complex) cysts can be difficult to accurately characterize and manage. ${ }^{15-17}$ Additionally, the number of nonsimple cysts is increasing, partly because of high-quality ultrasound imaging, which can now visualize small particulate matter in the cysts and which sometimes introduces imaging artifacts, such as reverberations, in their interiors. ${ }^{15,16}$ Because many patients have multiple cysts in each breast, needle aspiration, biopsy, or close followup cannot be performed on each cyst. Therefore, the ability to accurately diagnosis cysts with alternative imaging methods, such as elastography, becomes increasingly important.

A signal in fluid-filled cysts is caused by random noise, reverberations, side lobes, and clutter, which decorrelate rapidly under compression. Because cysts are not continuous media in the mechanical sense, the underlying hypotheses used in elastography do not hold. Therefore, speckle motion in cysts behaves differently compared with speckle motion in connected solid tissues: each speckle spot in a cyst moves independently of its neighbors. Even in complicated cysts, which have low-level internal echoes and fluid-debris levels, there is insufficient speckle for the speckle-tracking algorithms to reliably track motion between precompression and postcompression frames. In this study, all cysts were visible in the correlation coefficient images, with correlation coefficients $14 \%$ to $36 \%$ lower than those of the surrounding tissue. 
Thus, in theory, cysts could be strictly separated from solid masses on the basis of differences in their speckle-tracking correlation coefficients. Although these results are very preliminary, they suggest that one could pick at least an upper bound of the mean differences between the correlation coefficients in a lesion and background above which masses would, with near $100 \%$ specificity, be cysts. This would provide a very high-contrast imaging and detection method for cyst discrimination with very few false-positive results. This relationship would be true even for complex solid masses where the strains may be complex, but the speckle would still remain correlated on deformation.

In contrast, our cysts had a highly variable pattern in elastograms, and this variability in elastographic cyst patterns is consistent with previous literature results. ${ }^{8-10}$ Thus, elastograms of cysts can contain high strains, low strains, or any combination of the two. This is manifested as random black-and-white regions in our cyst elastograms (Figures 1-3), and this variability is reflected in the low signal-to-noise and contrastto-noise ratios in cysts (Table 1). Hence, it is nearly impossible to assign a characteristic elastographic pattern for a cyst because of the fact that the strain estimates are based on tracking noise.

Additionally, cysts in the correlation coefficient images in this study were sometimes smaller than in their corresponding gray scale ultrasound images because of partial volume averaging with the solid tissue near the boundaries. Thus, because of this solid material component in the tracked positions at the boundary, the correlation coefficients at the boundaries will be relatively high compared with the correlation coefficients inside cysts. Partial volume averaging, side lobes, and multiple scattering caused cyst edges to stay somewhat correlated between frames, creating the appearance that the cysts were somewhat smaller in the correlation coefficient images than in the gray scale images. This explains why the edges in the cysts can look brighter on the correlation coefficient images than the centers of the cysts. This finding might have clinical value because it is the reverse of cancers, which frequently look larger in elasticity images than in gray scale images. ${ }^{9}$
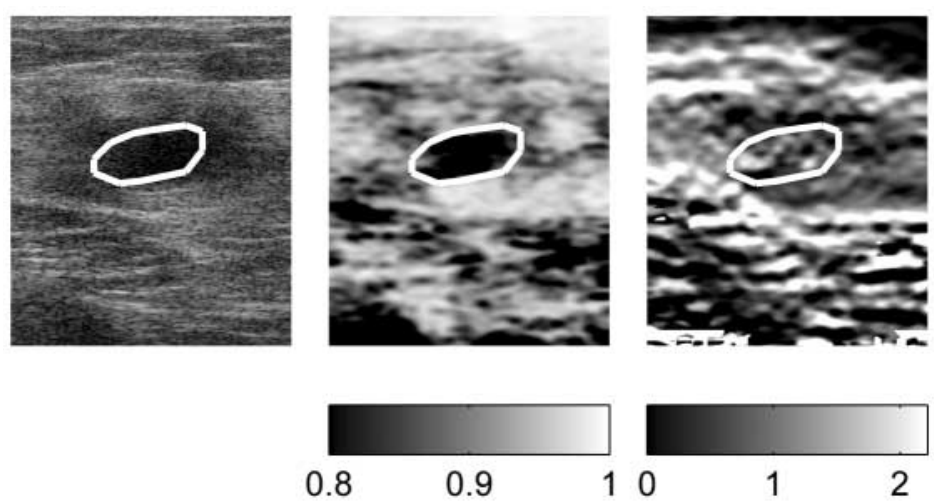

Figure 3. Gray scale image (left), correlation coefficient image (center), and elastogram (right) of a nonsimple cyst. Black regions in the correlation coefficient image correspond to areas of poor correlation; black regions in the elastogram correspond to areas of low strain (stiffer tissue). Strains are represented in percents. In this example, the area of the cyst in the elastogram contains both bright and dark areas with very little contrast between the cyst and the background. The variable bright and dark areas are due to the fact that noise is being used to calculate the strain images $[S / N(\varepsilon)=1.4]$, yet the mean correlation coefficient in the cyst is 0.75 , which is $17 \%$ lower than the background correlation of 0.90 . Hence, $\operatorname{S} / N(\rho)=10.7$ permits the cyst to be easily visualized. Original image dimensions were $38 \times 26 \mathrm{~mm}$.

An additional advantage to using correlation coefficient images rather than elastograms for cyst diagnosis is that correlation coefficients should be largely independent of preload. Preload can reduce contrast in elastograms of solid tissues because of the nonlinearity of tissue elasticity. ${ }^{9}$ Because of this nonlinearity, contrast between solid masses and background will decrease at higher preloads. ${ }^{18-21}$ Thus, maximum strain contrast between masses and background occurs at near-zero preloads. However, the signal in a cyst either is noise or moves randomly when compressed, independent of the level of precompres-

Table 1. Comparison of the Strain Properties in 4 Cysts With Their Correlation-Tracking Properties

\begin{tabular}{lcccc}
\hline Cyst & $\boldsymbol{S} / \boldsymbol{N}(\boldsymbol{\varepsilon})$ & $\boldsymbol{S} / \boldsymbol{N}(\rho)$ & $\boldsymbol{C} / \boldsymbol{N}(\boldsymbol{\varepsilon})$ & $\boldsymbol{C} / \boldsymbol{N}(\rho)$ \\
\hline 1 & 0.52 & 3.33 & 0.03 & 1.41 \\
2 & 1.36 & 10.7 & 0.15 & 2.14 \\
3 & 1.15 & 11.3 & 0.20 & 1.86 \\
4 & 0.34 & 3.16 & 0.05 & 2.37 \\
\hline
\end{tabular}

$S / N(\varepsilon)$ and $C / N(\varepsilon)$ are the strain signal-to-noise and contrast-to-noise ratios, respectively, whereas $S / N(\rho)$ and $C / N(\rho)$ are the correlation coefficient signal-to-noise and contrast-to-noise ratios. The correlation coefficient $S / N$ is at least 5 times greater than the strain $S / N$, and the correlation coefficient $C / N$ is at least 9 times greater than the strain $C / N$. These differences indicate that correlation coefficient images of cysts may be more robust and higher quality than their corresponding strain images. 

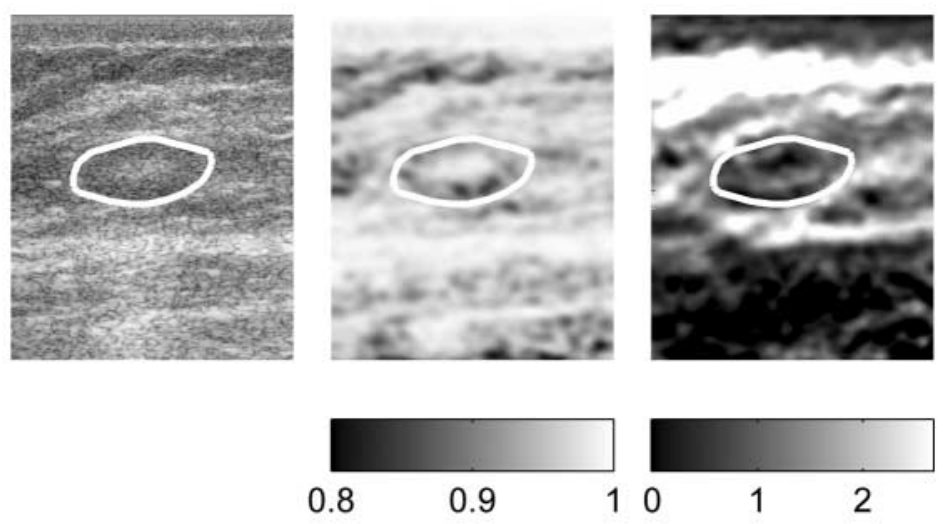

Figure 4. Gray scale image (left), correlation coefficient image (center), and elastogram (right) of benign breast parenchyma. Black regions in the correlation coefficient image correspond to areas of poor correlation; black regions in the elastogram correspond to areas of low strain (stiffer tissue). Strains are represented in percents. In direct contrast to the highly decorrelating cysts, the mean correlation coefficient in this solid mass is 0.94 , which is only $2.1 \%$ lower than the tissue directly surrounding it. Original image dimensions were $38 \times 26 \mathrm{~mm}$.

sion, and speckle tracking in cysts should be poor in all cases. Hence, there should be no minimum loading requirement for distinguishing cysts from solid masses as long as one is still able to deform the tissue. Thus, cysts should always look different from background solid material on correlation coefficient images no matter what the preload. This relationship between tracking correlation coefficients in cysts and background solid material should hold for tracking in 1, 2, or 3 dimensions and should be independent of the direction of deformation. Future studies will explore this hypothesis further.

In summary, although our very preliminary study of only 4 cyst cases precludes statistical comparisons, these results suggest that using correlation coefficient images to identify cysts with 2D elastography should produce more consistent results than using elastograms. These successes are achieved by using the low signal levels and relatively unrestricted motion of the scatterers in fluid-filled cysts, which decorrelate much more quickly than surrounding tissue when compressed. Hence, it is not surprising that elastograms of cysts are very noisy, making it possible for cysts to have almost any appearance. This explains the diverse appearances of cysts in elastograms using different equipment and processing. ${ }^{8-11}$
Future studies should compare the correlation coefficient images between cysts and hypoechoic solid masses, which could also decorrelate quickly if they have insufficient signal-to-noise ratios for speckle tracking. However, these preliminary results suggest that the correlation coefficient image contains information that could aid cyst/solid mass discrimination.

\section{References}

1. Chen X, Li X, Sahn D, Kim K, Xie H, O’Donnell M. Evaluation of 2-D speckle tracking based strain rate imaging (SRI) using a 3-D heart simulation model. IEEE Ultrason Symp Proc 2004; 3:2125-2128.

2. Céspedes El, de Korte CL, van der Steen AFW, von Birgelen C, Lancée CT. Intravascular elastography: principle and potentials. Semin Interv Cardiol 1997; 2:55-62.

3. de Korte CL, Pastercamp G, van der Steen AFW, Woutman HA, Bom N. Characterization of plaque components with intravascular ultrasound elastography in human femoral and coronary arteries in vitro. Circulation 2000; 102: 617-623.

4. Weitzel WF, Kim K, Rubin JM, Xie H, O'Donnell M. Renal advances in ultrasound elasticity imaging: measuring the compliance of arteries and kidneys in end-stage renal disease. Blood Purif 2005; 23:10-17.

5. Rubin JM, Xie H, Kim K, et al. Sonographic elasticity imaging of acute and chronic deep venous thrombosis in humans. J Ultrasound Med 2006; 25:1179-1186.

6. Lorenz A, Sommerfield HJ, Garcia-Schurmann MG, Philippou S, Senge T, Ermert H. A new system for the acquisition of ultrasonic multi-compression strain images of human prostate in vivo. IEEE Trans Ultrason Ferroelectr Freq Control 1999; 46:1147-1154.

7. Pesavento A, Lorenz A, Siebers S, Ermert H. New real-time strain imaging concepts using diagnostic ultrasound. Phys Med Biol 2000; 45:1423-1435.

8. Garra BS, Céspedes El, Ophir J, et al. Elastography of breast lesions: initial clinical results. Radiology 1997; 202:79-86.

9. Hall TJ, Zhu Y, Spalding CS. In vivo real-time freehand palpation imaging. Ultrasound Med Biol 2003; 29:427-435.

10. Barr RG. Clinical applications of a real time elastography technique in breast imaging. In: Proceedings of the Fifth International Conference on the Ultrasonic Measurement and Imaging of Tissue Elasticity; October 2006; Snowbird, UT; 112 .

11. Lyshchik A, Higashi $T$, Asato $R$, et al. Thyroid gland tumor diagnosis at US elastography. Radiology 2005; 237:202211

12. Kaluzynski K, Chen X, Emelianov S, Skovoroda A, O'Donnell M. Strain rate imaging using two-dimensional speckle tracking. IEEE Trans Ultrason Ferroelectr Freq Control 2001; 48:1111-1123. 
13. Lubinski M, Emelianov S, O'Donnell M. Speckle tracking methods for ultrasonic elasticity imaging using short-time correlation. IEEE Trans Ultrason Ferroelectr Freq Control 1999; 46:82-96.

14. O'Donnell M, Skovoroda AR, Shapo BM, Emelianov SY Internal displacement and strain imaging using ultrasonic speckle tracking. IEEE Trans Ultrason Ferroelectr Freq Control 1994; 41:314-325

15. Stavros AT, Thickman D, Rapp CL, Dennis MA, Parker SH, Sisney GA. Solid breast nodules: use of sonography to distinguish between benign and malignant lesions. Radiology 1995; 196:123-134.

16. Venta LA, Kim JP, Pelloski CE, Morrow M. Management of complex breast cysts. AJR Am J Roentgenol 1999; 173: $1331-1335$

17. Berg WA, Campassi $\mathrm{Cl}$, loffe OB. Cystic lesions of the breast: sonographic-pathologic correlation. Radiology 2003; 227:183-191.

18. Krouskop TA, Wheeler TM, Kallel F, Garra BS, Hall T. Elastic moduli of breast and prostate tissues under compression. Ultrason Imaging 1998; 20:260-274.

19. Erkamp RQ, Emelianov SY, Skovoroda AR, O'Donnell M. Nonlinear elasticity imaging: theory and phantom study. IEEE Trans Ultrason Ferroelectr Freq Control 2004; 51:532-539.

20. Erkamp RQ, Emelianov SY, Skovoroda AR, O'Donnell M. Measuring the nonlinear elastic properties of tissue-like phantoms. IEEE Trans Ultrason Ferroelectr Freq Control 2004; 51:410-419.

21. Emelianov SY, Erkamp RQ, Lubinski MA, Skovoroda AR, O'Donnell M. Non-linear tissue elasticity: adaptive elasticity imaging for large deformations. IEEE Ultrason Symp Proc 1998; 2:1753-1756. 